\title{
On the origin of highest energy gamma-rays from Mkn 501
}

\author{
F. A. Aharonian ${ }^{1}$, A. N. Timokhin ${ }^{1,2}$, and A. V. Plyasheshnikov ${ }^{1,3}$ \\ 1 Max-Planck-Institut für Kernphysik, Saupfercheckweg 1, 69117, Heidelberg, Germany \\ 2 Sternberg Astronomical Institute, Universitetskij pr. 13, 119899, Moscow, Russia \\ 3 Altai State University, Dimitrov Street 66, 656099 Barnaul, Russia
}

Received 27 August 2001 / Accepted 11 January 2002

\begin{abstract}
The spectra of very high energy $\gamma$-radiation from distant extragalactic objects suffer significant deformations during the passage of primary $\gamma$-rays through the intergalactic medium. The recently reported fluxes of diffuse infrared background radiation indicate that we detect, most probably, heavily absorbed TeV radiation from Mkn 421 and Mkn 501. This implies that the absorption-corrected spectrum of Mkn 501 may contain a sharp pile-up which contradicts to the predictions of the conventional models of TeV blazars, and thus may lead to the so-called "IR background-TeV gamma-ray crisis". To overcome this difficulty, in this paper we propose two independent hypotheses assuming that (i) the $\mathrm{TeV}$ radiation from Mkn 501 has a secondary origin, i.e. it is formed during the development of electron-photon cascades in the intergalactic medium initiated by primary $\gamma$-rays; (ii) the pile-up in the source spectrum is a result of comptonization (in deep Klein-Nishina regime) of ambient optical radiation by an ultrarelativistic conical cold outflow (jet) with bulk motion Lorentz factor $\Gamma_{0} \geq 3 \times 10^{7}$. Within the uncertainties caused by the limited energy resolution of spectral measurements, the observed TeV radiation of Mkn 501 formally can be explained by the intergalactic cascade $\gamma$-rays, assuming however an extremely low intergalactic magnetic field in the direction to the source at the level of $\leq 10^{-18} \mathrm{G}$. We also demonstrate that the "bulk motion comptonization" scenario can quite naturally reproduce the unusual spectral features in the absorption-corrected $\mathrm{TeV}$ spectrum of $\mathrm{Mkn} 501$, and briefly discuss the astrophysical implications of this hypothesis.
\end{abstract}

Key words. galaxies: BL Lacertae objects: individual: Mkn 501 - cosmology: diffuse radiation gamma rays: observations - gamma rays: theory

\section{Introduction}

The Diffuse Extragalactic Background Radiation (DEBRA) at infrared to ultraviolet wavelengths carries crucial cosmological information about the galaxy formation epochs. It is believed that this radiation basically consists of two emission components produced by stars and partly absorbed and re-emitted by dust during the entire history of evolution of galaxies. Consequently, two distinct bumps in the spectrum of red-shifted radiation at near infrared (NIR) $\lambda \sim 1-2 \mu \mathrm{m}$ and far infrared (FIR) $\lambda \sim 100-200 \mu \mathrm{m}$ wavelengths, and a mid infrared (MIR) "valley" between these bumps are expected (see e.g. Dwek et al. 1998; Primack et al. 1999; Pei et al. 1999; Silk \& Devriendt 2000; Malkan \& Stecker 200; Hauser \& Dwek 2001; Franceschini et al. 2001).

Direct measurements of the Cosmic Infrared Background (CIB) radiation contain large uncertainties because of heavy contamination caused by foregrounds

Send offprint requests to: F. A. Aharonian,

e-mail: Felix.Aharonian@mpi-hd.mpg.de of different origin (for review see Hauser \& Dwek 2001). Gamma-ray astronomy offers a complementary approach to derive information about CIB. Although this method requires certain model assumptions about the primary (un-absorbed) spectrum of $\gamma$-rays, it has an adequate potential for robust conclusions concerning the absolute flux and the spectrum of CIB. Moreover, the study of angular and spectral properties of $\gamma$-radiation from predicted giant electron-positron halos surrounding powerful nonthermal extragalactic objects like AGN and radiogalaxies with known redshifts (Aharonian et al. 1994) can provide a unique tool to "measure" unambiguously the broad-band spectrum and the absolute flux of CIB at different cosmological epochs, and thus to probe the evolution of galaxies in past.

The spectra of high energy $(E \geq 10 \mathrm{GeV}) \gamma$-radiation observed from distant extragalactic objects suffer significant deformation during their passage through the intergalactic medium due to interactions of primary $\gamma$-rays with CIB (Nikishov 1962; Gould \& Schreder 1967; Stecker et al. 1992). The absorption features in the $\gamma$-ray spectra 
depends on the flux of CIB, thus the study of such features from extragalactic objects with firmly established distances could yield important constraints on CIB. Strictly speaking, this approach requires good understanding of the source spectra of $\gamma$-rays from ensemble of sources located at different cosmological distances. Otherwise, the conclusions based merely on $\gamma$-ray observations from a single source would be essentially model-dependent, and therefore would permit different interpretations concerning both the intrinsic $\gamma$-ray spectrum, and the flux of CIB.

Presently we do face such an ambiguity, when trying to interpret the multi-TeV $\gamma$-ray emission of Mkn 501 observed during its remarkably strong and long flare in 1997 (Aharonian et al. 1999b). The only definite conclusion which can be drawn from these observations is that we see, most probably, significantly absorbed $\mathrm{TeV}$ radiation, especially at energies above $10 \mathrm{TeV}$, for which the optical depth could be as large as 10 (Coppi \& Aharonian 1999b; Finkbeiner et al. 2000; Protheroe \& Meyer 2000). Moreover, a non-negligible absorption may take place already at low, sub-TeV energies (Coppi \& Aharonian 1999b; Guy et al. 2000). It should be noticed that the analysis of the intergalactic absorption at sub-TeV and multi-TeV parts of the spectrum of Mkn 501 leads to two essentially different conclusions. The absorption-corrected $\gamma$-ray spectrum at low energies, based on the CIB fluxes reported at 2.2 and $3.5 \mu \mathrm{m}$ (Dwek \& Arendt 1998; Gorjian et al. 2000; Wright \& Johnson 2001) and on the current theoretical predictions for NIR (see e.g. Primack et al. 2001), is in a general agreement with the Synchrotronself-Compton (SSC) model of $\mathrm{X}$ - and $\mathrm{TeV}$ radiation of Mkn 501 (see e.g. Guy et al. 2000; Krawczynski et al. 2000; Primack et al. 2001). On the other hand, the corrections to the $\gamma$-ray spectrum at energies above $10 \mathrm{TeV}$ based on the unexpectedly large CIB fluxes detected by COBE at 140 and $240 \mu \mathrm{m}$ (Hauser et al. 1998; Schlegel et al. 1998; Lagache et al. 1999) result in an "unreasonable" source spectrum. Namely it implies a source spectrum which sharply curves up above $10 \mathrm{TeV}$, unless we assume that the CIB flux at MIR between 10 and $50 \mu \mathrm{m}$ is quite low (a few $\mathrm{nW} / \mathrm{m}^{2} \mathrm{sr}$ ), and at longer wavelengths it increases rapidly $\left(\nu F_{\nu} \propto \lambda^{s}\right.$ with $\left.s \geq 2\right)$ in order to match the COBE points (Aharonian et al. 1999b; Coppi \& Aharonian 1999b; Renault et al. 2001). Even so, the large DEBRA fluxes at 140 and $250 \mu \mathrm{m}$ imply very flat "reconstructed" source $\gamma$-ray spectral energy distributions (SED), $\nu S_{\nu}=E^{2} \mathrm{~d} N / \mathrm{d} E=E^{2-\alpha}$ with a photon index $\alpha \leq 2$. Such flat source spectra extending beyond $10 \mathrm{TeV}$ require, within SSC models, rather unconventional jet parameters, namely very large Doppler factors and very small magnetic fields (H. Krawczynski and P. Coppi, private communication; J. Kirk, private communication).

A real trouble arises, however, when we take into account the recent claims about detection of CIB flux at $100 \mu \mathrm{m}$ (Lagache et al. 1999; Finkbeiner et al. 2000), and especially at $60 \mu \mathrm{m}$ (Finkbeiner et al. 2000). If we refer the reported fluxes to the truly diffuse extragalactic background radiation, then a little room would be left for speculations concerning the spectral shape of CIB in order to prevent the "unreasonable" $\gamma$-ray source spectrum. This implies that we should accept the existence of a sharp pile-up in the spectrum of $\mathrm{TeV}$ radiation. Motivated by such a non-standard spectral shape of TeV-radiation, recently several extreme assumptions have been made in order to overcome the "IR background-TeV gamma-ray crisis" (Protheroe \& Meyer 2000). In particular, Harwit et al. (1999) suggested an interesting hypothesis that the HEGRA highest energy events are due to Bose-Einstein condensations interacting with the air atmosphere, and proposed a test to inspect this hypothesis by searching for peculiar features of showers detected by HEGRA in the direction of Mkn 501. Subsequently, the HEGRA collaboration has demonstrated (Aharonian et al. 2000) that the detected shower characteristics are in fact in good agreement with the predictions for the events initiated by ordinary $\gamma$-rays. Another, even more dramatic hypothesis violation of the Lorentz invariance - has been proposed by several authors (see e.g. Coleman \& Glashow 1999; Kifune 1999; Kluźniak 1999; Aloisio et al. 2000; Amelino-Camelia \& Piran 2001; Protheroe \& Meyer 2000) to solve this problem. We may add to the list of "exotic" solutions of the "IR background-TeV gamma-ray crisis" a less dramatic, in our view, hypothesis, assuming that Mkn 501 is located at a distance significantly less than $100 \mathrm{Mpc}$ - good news for the advocates of non-cosmological origin of some of AGN and quasars (see e.g. Hoyle \& Burbidge 1996; Arp et al. 1997).

Although very fascinating, it seems to us too premature to invoke such dramatic revisions of essentials of modern physics and astrophysics. The nature of the FIR isotropic emission detected by COBE is not yet firmly established, and it is quite possible that the bulk of the reported flux, especially below $100 \mu \mathrm{m}$, is a result of superposition of different local backgrounds. Needless to say, that this would be the simplest solution of the problem (Stecker 2000; Renault et al. 2001). In this paper we adopt, however, that the reported FIR fluxes have universal (extragalactic) origin. This implies that we adopt the existence of a pronounced pile-up in the $\gamma$-ray spectrum of Mkn 501 above $10 \mathrm{TeV}$, but try to find an explanation of this spectral feature within the framework of new but yet conventional astrophysical scenarios. In this paper we propose and study two potential ways to overcome the "IR background-TeV gamma-ray crisis":

(1) $\mathrm{TeV} \gamma$-rays from Mkn 501 are not direct representatives of primary radiation of the source, but have a secondary origin, i.e. they are formed during the development of high energy electron-photon cascades in the intergalactic medium initiated by interactions of primary $\gamma$-rays with diffuse extragalactic photons. This hypothesis implies a rather extreme assumption concerning the strength of the intergalactic magnetic field on $\geq 1 \mathrm{Mpc}$, $B \leq 10^{-18} \mathrm{G}$.

(2) The "reconstructed" source spectrum of Mkn 501 with a flat (almost constant) SED below $10 \mathrm{TeV}$, and a pile-up beyond $10 \mathrm{TeV}$, is partly or entirely produced 


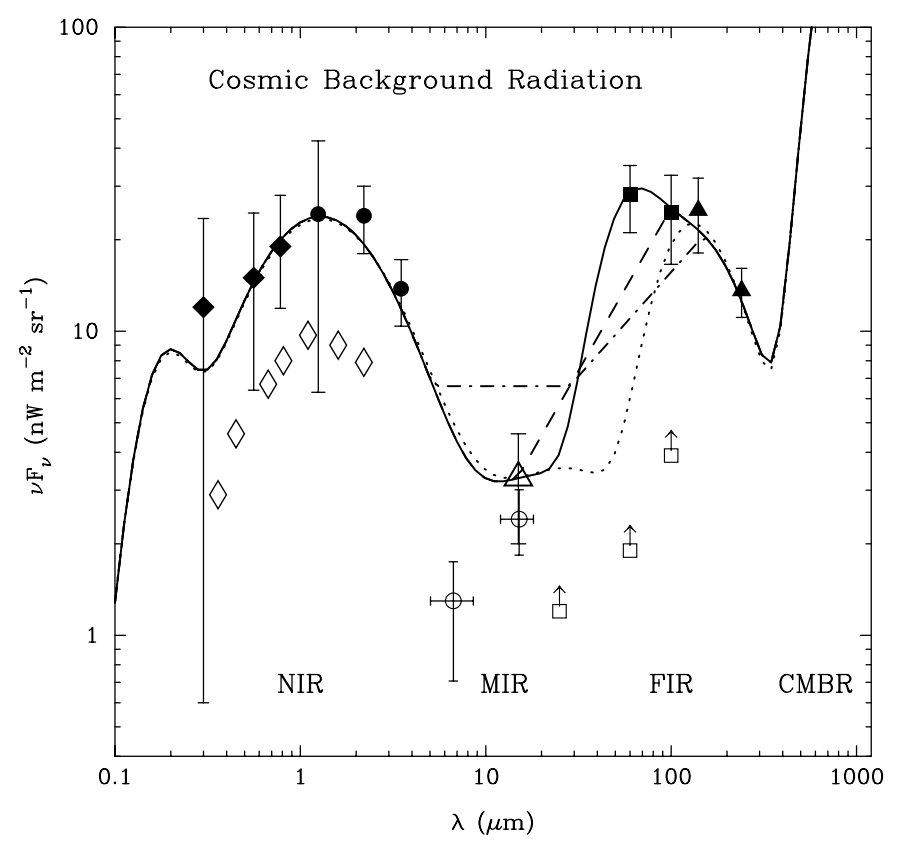

Fig. 1. Cosmic background radiation. The reported fluxes are shown with filled symbols: Bernstein (1999) - diamonds, Wright \& Johnson (2001) - circles, Finkbeiner et al. (2000) squares, Hauser et al. (1998) - triangles. The low limits are shown by open symbols: Pozzetti et al. (1998) - diamonds, Biviano et al. (2000) - triangle, Franceschini et al. (2001) circles, Hacking \& Soifer (1991) - squares, The CIB models are shown by solid line - Model I, by dotted line - Model II, by dashed line - Model III, by dot-dashed line - Model IV (for details see the text).

by monoenergetic ultrarelativistic beam of electrons due to the inverse Compton scattering in deep Klein-Nishina regime. In order to avoid significant radiative (synchrotron and Compton) losses, which otherwise would result in an equilibrium, $E_{\mathrm{e}}^{-2}$ type differential spectrum of electrons, we assume that such a beam of electrons in fact is a cold, conical kinetic-energy dominated ultrarelativistic wind with the bulk Lorentz factor $\Gamma_{0} \sim 4 \times 10^{7}$ formed beyond the accretion disk of the central black hole. Apparently this hypothesis implies non-acceleration origin of highest energy $\gamma$-rays detected from Mkn 501.

\section{Absorption of gamma-rays in $\mathrm{CIB}$}

The reported fluxes and flux upper/low limits of CIB from optical/UV to far IR wavelengths are shown in Fig. 1. The reliability and the implications of these measurements are discussed in the recent review article by Hauser \& Dwek (2001). The level of the spectral energy distribution (SED) of CIB at optical/NIR wavelengths with the "best guess estimate" between 20 and $50 \mathrm{nW} / \mathrm{m}^{2} \mathrm{sr}$ is comparable with the overall energy flux of FIR of about $40-50 \mathrm{nW} / \mathrm{m}^{2} \mathrm{sr}$ (Pozzetti \& Madau 2000). This indicates that an essential part of the energy radiated by stars is absorbed and reemitted by dust in a form of thermal sub-mm emission. Currently the information at mid-infrared wavelengths is very limited. The only available measurement at 6 and
$15 \mu \mathrm{m}$ in Fig. 1 derived from the ISOCAM source counts (Franceschini et al. 2001) shown in Fig. 1 should be treated as a lower limit. In Fig. 1 we show also a slightly higher flux estimate at $15 \mu \mathrm{m}$ reported by Biviano et al. (2000). Therefore the flux estimate at the level of $\simeq 2-3 \mathrm{nW} / \mathrm{m}^{2}$ sr (Franceschini et al. 2001) as well as the lower limits based on the IRAS counts at 25-100 $\mu \mathrm{m}$ (Hacking \& Soifer 1991) do not allow firm conclusions about the depth of the MIR "valley" dominated by radiation of the warm dust component. Consequently, it does not provide sufficient information for definite predictions regarding the slope of the spectrum in the most crucial (from the point of view of absorption of $\geq 10 \mathrm{TeV} \gamma$-rays) MIR-to-FIR transition region.

As long as the available measurements of CIB do not allow a quantitative study of the effect of absorption of $\gamma$-rays in the intergalactic medium, we can rely only on model predictions or on the "best guess" shape of the CIB spectrum. In this regard we notice that the reported high FIR fluxes present a common problem for all current CIB models. Therefore, if one adopts that the reported FIR fluxes have truly diffuse extragalactic origin, an essential revision of the CIB models is needed in order to match the data. Such an attempt has been made recently by Primack et al. (2001) who showed that their semi-analytical approach, with a reasonable adjustment of some model parameters, and using the empirical dust emission templates of Dwek et al. (1998), can match the reported FIR fluxes.

In Fig. 1 we show several model spectra of CIB. Since we are interested, first of all, in the cosmic background fluxes at MIR and FIR, at shorter wavelengths we adopt a common for all models approximation which matches the reported optical and NIR fluxes. The smooth template of the CIB spectrum in the most principal MIR-to-FIR transition region shown by dashed line (hereafter Model III) in Fig. 1, fits the reported fluxes including, within $2 \sigma$ uncertainty, the $60 \mu \mathrm{m}$ point. Actually this idealized template is quite similar to the CIB spectrum shown in Fig. 1 of Franceschini et al. (2001) based on their reference model for IR galaxy evolution, as well as to some of the recent theoretical models of Primack et al. (2001). It has a rather flat shape in the MIR-to-FIR transition region, $\nu F_{\nu} \propto \lambda^{s}$ with $s \leq 1$. This results in short mean free paths of $\gamma$-rays above $10 \mathrm{TeV}$ ( $L \leq 50 \mathrm{Mpc}$; see Fig. 2 ), and consequently in a pile-up in the reconstructed $\gamma$-ray source spectrum (see Fig. 3) defined as:

$J_{0}(E)=J_{\text {obs }}(E) \exp [\tau(E)]$,

where $J_{\text {obs }}$ is the observed $\gamma$-ray spectrum, $\tau(E)=d / L(E)$ is the intergalactic optical depth, and $d$ is the distance to the source.

If we adopt that the reported FIR fluxes correctly describe the level of truly diffuse background radiation, only two ways are left for reduction of the effect of attenuation of $\geq 10 \mathrm{TeV} \gamma$-rays: (i) an ad hoc assumption of the CIB flux at wavelengths between 10 and $60 \mu \mathrm{m}$ at the marginally acceptable (i.e. the ISOCAM low-limit) level, but with very rapid rise beyond $60 \mu \mathrm{m}$ in order 


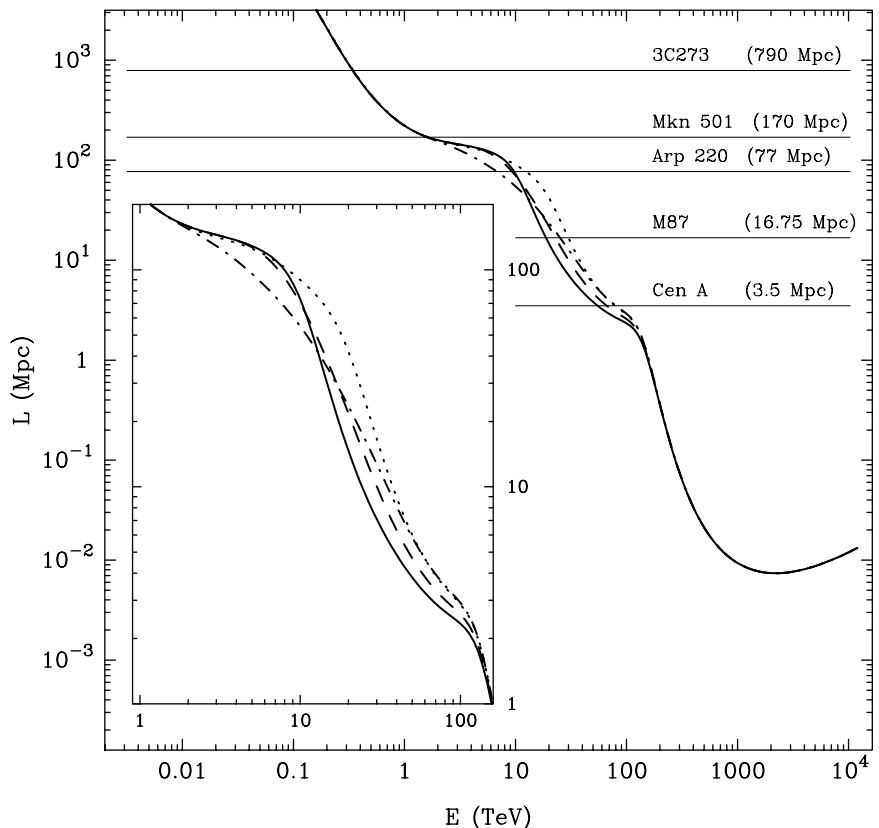

Fig. 2. Mean free path of $\gamma$-rays for 4 different models of the CIB spectrum: solid line - Model I, dotted line - Model II, dashed line - Model III, dot-dashed line - Model IV. The horizontal lines indicate the distances to Cen A, M87, Mkn 501, and $3 \mathrm{C} 273\left(H_{0}=60 \mathrm{kms}^{-1} \mathrm{Mpc}\right)$.

to match the reported fluxes at FIR, and (ii) adopting for the Hubble constant $H_{0} \simeq 100 \mathrm{~km} \mathrm{~s}^{-1} \mathrm{Mpc}$, i.e. assuming the smallest possible distance to Mkn 501 $d=c z / H_{0}=102 \mathrm{Mpc}(z=0.034)$.

In Fig. 1 we show 2 other model spectra of CIB (solid line - Model I, dotted line - Model II) which fit the data at NIR and FIR, but at the same time allow minimum intergalactic $\gamma$-ray absorption at $E \geq 10 \mathrm{TeV}$, because both spectra are forced to be at the lowest possible level at MIR-to-FIR transition region set by the ISOCAM lower limit at $15 \mu \mathrm{m}$.

The CIB fluxes above $140 \mu \mathrm{m}$ are approximated by the function suggested by Fixsen et al. (1998). Below $140 \mu \mathrm{m}$ for both I and II Models we assume the same spectral shape described by Planckian distribution. Such spectra should be considered as a lower limit, because in the most critical MIR-FIR transition region near $60 \mu \mathrm{m}$ it is essentially the Wien part of the blackbody spectrum - the steepest (physically justified) continuous spectrum. The Model II marginally agrees with the $100 \mu \mathrm{m}$ point but underestimates the flux at $60 \mu \mathrm{m}$ by a factor of 5 compared with the flux reported by Finkbeiner et al. (2000). This model significantly suppresses the photon density between 50 and $100 \mu \mathrm{m}$, and correspondingly allows larger mean free paths for $\gamma$-rays with energy more than several $\mathrm{TeV}$ (Fig. 2). Such a SED of CIB results in almost $E^{-2}$ type "reconstructed" spectrum of $\gamma$-rays from Mkn 501 at energies between approximately 2 and $20 \mathrm{TeV}$ (Fig. 3). This has a simple explanation. For the constant SED of CIB (the flat part of the spectrum 2 in Fig. 1), $n_{\mathrm{CBR}}(\epsilon) \propto \epsilon^{-2}$, the photon-photon optical depth is proportional to $E$ with

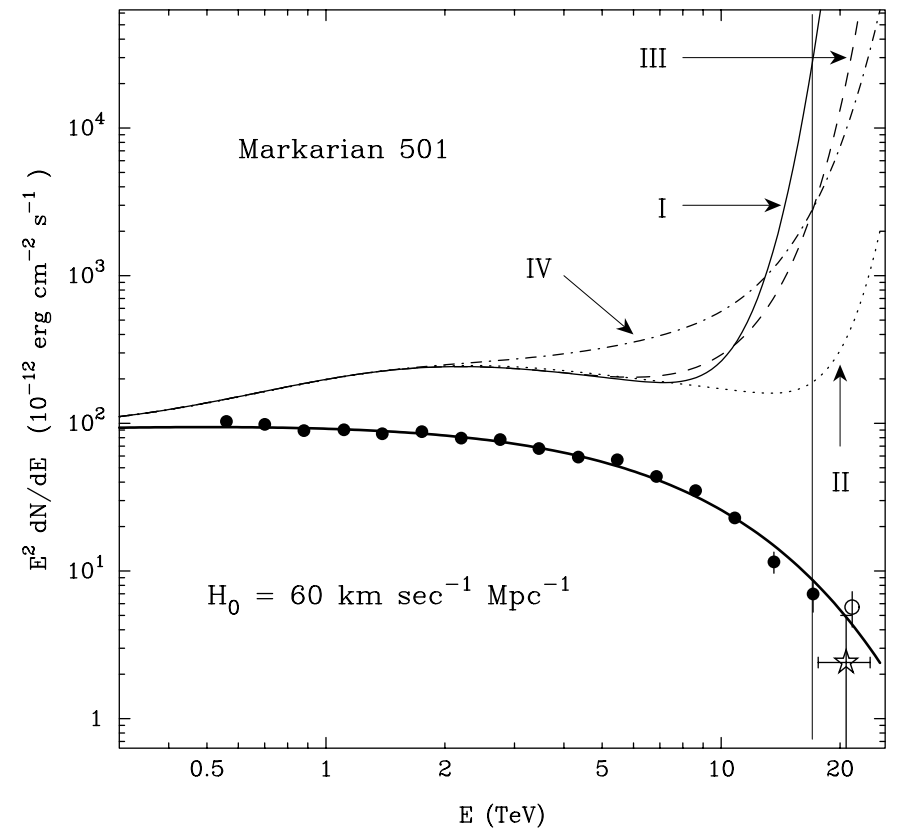

Fig. 3. Spectral Energy Distribution (SED) of Mkn 501. The experimental points (filled circles) correspond to the timeaveraged spectrum of Mkn 501 during the flare in 1997 (Aharonian et al. 1999b). The heavy line corresponds to the fit to this data in the form of Eq. (2). The star correspond to the flux in the highest energy bin around $21 \mathrm{TeV}$ obtained after the reanalysis of the same data set but with improved energy resolution (Aharonian et al. 2001b). The vertical line at $17 \mathrm{TeV}$ indicates the edge of the spectrum of Mkn 501 measured by HEGRA with high statistical significance. The solid, dotted, dashed and dot-dashed lines represent the reconstructed (absorption-corrected) spectra of $\gamma$-rays for the CIB Models I, II, III and IV, respectively $\left(H_{0}=60 \mathrm{~km} \mathrm{~s}^{-1} \mathrm{Mpc}\right)$.

an absolute value for $\mathrm{Mkn} 501 \tau(E) \approx 0.16(E / 1 \mathrm{TeV})$ (Aharonian et al. 1999b). Therefore, in the absorptioncorrected (source) spectrum of $\gamma$-rays given by Eq. (1), the optical depth $\tau$ almost compensates the exponential term of the observed spectrum of $\mathrm{Mkn} 501^{1}$, which from $0.5 \mathrm{TeV}$ to $20 \mathrm{TeV}$ can be presented in a simple "power-law with exponential cutoff" form (Aharonian et al. 1999b)

$J_{\mathrm{obs}}(E) \propto E^{-1.92} \exp (-E / 6.2 \mathrm{TeV})$.

Recently, the HEGRA collaboration published the results of reanalysis of the spectrum of Mkn 501 (Aharonian et al. 2001b) using an improved method of shower energy reconstruction with 10 to 12 per cent energy resolution (Hofmann 2000). Apparently the improved energy resolution is more relevant for derivation of the shapes of steep $\gamma$-ray spectra, e.g. spectra with exponential or sharper cutoffs. The new HEGRA spectral analysis confirmed the results of the previous study up to energy $17 \mathrm{TeV}$, but shows a steeper spectrum beyond that energy (Aharonian et al. 2001b). Therefore in Fig. 1 we show only

\footnotetext{
${ }^{1}$ Note that somewhat different CIB models with more complex spectral shapes can also lead to flat, $E^{-2}$ type powerlaw absorption-corrected $\gamma$-ray spectra (see e.g. Coppi \& Aharonian 1999b; Konopelko et al. 1999; Guy et al. 2000).
} 
one point of the new analysis - the flux in the highest energy bin around $21 \mathrm{TeV}$ (star) which lies significantly below the flux estimate of the previous study (open circle). We should notice, however, that the points above $17 \mathrm{TeV}$ in both papers do not have high statistical significance anyway, therefore below we limit our study only by the energy region up to $17 \mathrm{TeV}$ (i.e. by the region on the left side of the vertical line shown in Fig. 3). In this energy interval the energy spectra obtained by new and old methods are perfectly described (Aharonian et al. 2001b) by Eq. (2).

Apparently, for conventional $\gamma$-ray production mechanisms the $E^{-2}$ type $\gamma$-ray source spectrum sounds more comfortable than the spectrum containing a sharp pileup. Therefore, the Model II in Fig. 1 can be treated as the "most favorable" CIB spectrum, although its flat shape in the broad MIR-to-FIR transition region with very fast increase beyond $60 \mu \mathrm{m}$ hardly match the current theoretical descriptions of CIB, as well as the FIR spectra observed from nearby galaxies. Such a comparison is of special interest, especially because the bulk of the integrated extragalactic background radiation has been generated presumably at $z<1$ (Harwit 1999; Franceschini et al. 2001). In particular, the shape of the Model II contradicts to the average SED of the ISOCAM sources which not only contribute a dominant fraction to CIB at MIR, but likely are major contributors at longer wavelengths as well (Franceschini et al. 2001).

All current CIB models have a problem to accommodate the reported flux at $60 \mu \mathrm{m}$. If this point, however, is the representative of the truly diffuse flux, we must assume a CIB spectrum close to the Model I shown in Fig. 1 by the solid line. This Model assumes a very steep slope between 30 and $60 \mu \mathrm{m}$. A steeper spectrum (i.e. steeper than the Wien tail of the black-body radiation) in this narrow wavelength band hardly could be physically justified. Moreover, already $60 \mu \mathrm{m}$ photons have sufficient energy for effective interaction with $\geq 10 \mathrm{TeV} \gamma$-rays, therefore we cannot suppress anymore the severe $\gamma$-ray absorption by speculating about the spectral shape of CIB (see Fig. 4 and the related discussion below).

Finally, in Fig. 1 we show one more possible model spectrum of CIB (dot-dashed curve - Model IV) which assumes significantly, by a factor of 2.5 , higher flux at $15 \mu \mathrm{m}$ compared to the reported ISOCAM lower, and smoothly passes through the low edges of the error bars of reported fluxes at 100 and $140 \mu \mathrm{m}$. Surprisingly such a high at MIR spectrum does not result in an unusual $\gamma$-ray spectrum as long as it concerns the energy region below $10 \mathrm{TeV}$. Namely, at these energies we obtain an almost single hard power-law $\gamma$-ray source spectrum with photon index less than 2. Above $10 \mathrm{TeV}$ we again observe a pile-up which however in this case is less pronounced than in the case of Models I and III.

A $\gamma$-ray photon with energy $E$ propagating trough isotropic photon field can interact, via electron-positron pair production, with ambient photons of energy $\epsilon \geq \epsilon_{\mathrm{th}}=$ $\left(m_{\mathrm{e}} c^{2}\right)^{2} / E \simeq 0.26\left(E_{\gamma} / 1 \mathrm{TeV}\right)^{-1} \mathrm{eV}$ or of wavelength

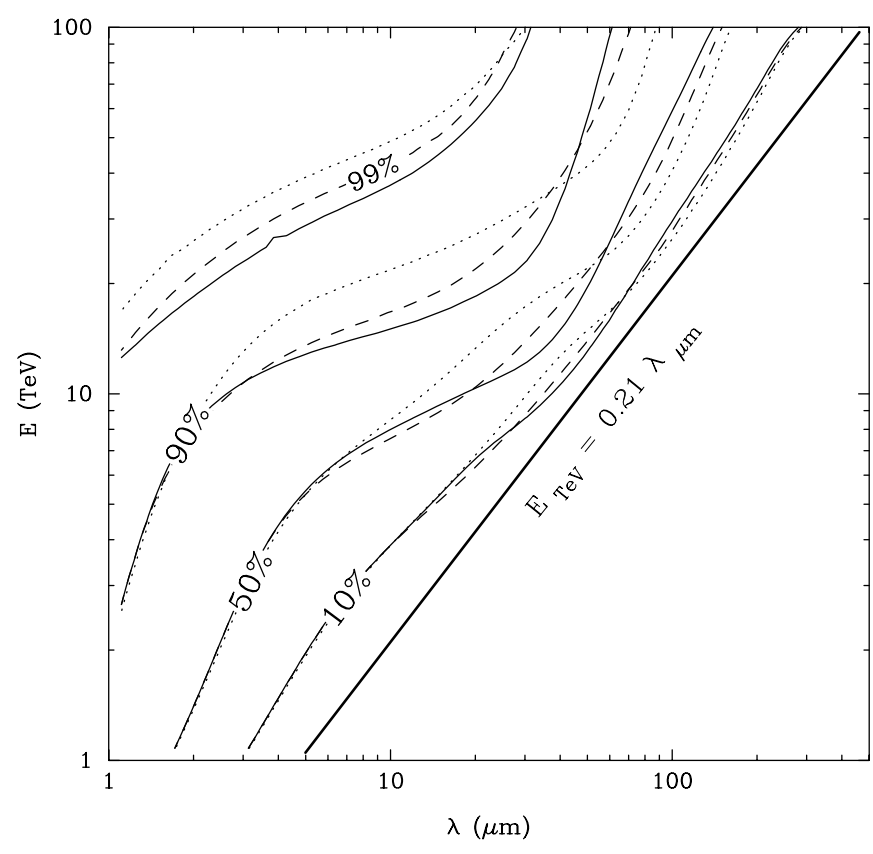

Fig. 4. The contour map of the function $\kappa(\lambda, E)$. The solid, dotted and dashed lines show the levels of $\kappa(\lambda, E)$ for the CIB Models I, II, and III, respectively. The heavy solid line represents the threshold of $\gamma \gamma$ pair production.

$\lambda=4.8\left(E_{\gamma} / 1 \mathrm{TeV}\right) \mu \mathrm{m}$. The latter relation is represented in Fig. 4 by the solid line. The cross-section of $\gamma \gamma$ interactions averaged over the directions of background photons peaks at $\epsilon_{\max } \simeq 3.5 \epsilon_{\text {thresh }}$ with $\sigma_{\gamma \gamma}^{\max } \simeq(1 / 4) \sigma_{\mathrm{T}}$ (see, e.g. Vassiliev 2000)

Therefore, even for a broad, e.g. power-law spectrum of background radiation, the most contribution to the optical depth $\tau$ comes from a narrow spectral band of CIB within $\epsilon_{\max } \pm \Delta \epsilon$ with $\Delta \epsilon \sim 1 / 2 \epsilon_{\max }$. For typical spectra of CIB with two distinct NIR and FIR bumps and a MIR valley, the " $\gamma$-ray energy-CIB photon wavelength" relation is more complicated, namely the relative contributions of different spectral intervals of CIB to the optical depth $\tau_{0}$ significantly depend on the energy of the primary $\gamma$-ray photon. It is convenient to describe this dependence by the ratio $\kappa(E, \lambda)=\tau(E, \lambda) / \tau_{0}(E)$, where

$\tau(E, \lambda)=\int_{\lambda}^{\lambda_{\mathrm{th}}} \sigma_{\gamma \gamma}\left(E_{\gamma}, \lambda\right) n_{\mathrm{CBR}}(\lambda) \mathrm{d} \lambda$,

and $\tau_{0}=\tau\left(E_{\gamma}, \lambda=0\right)$ is the optical depth integrated over the entire spectral range of CIB $n_{\mathrm{CIB}}(\lambda)$ above the threshold $\lambda_{\text {th }} \simeq 4.8\left(E_{\gamma} / 1 \mathrm{TeV}\right) \mu \mathrm{m}$.

In Fig. 4 the contour map of the function $\kappa(E, \lambda)$ is shown for 3 different models of CIB in the spectral range of $\gamma$-rays from 1 to $100 \mathrm{TeV}$, and for the CIB photons from 1 to $500 \mu \mathrm{m}$. For the given energy of $\gamma$-ray photon $E_{0}$, the spectral region of CIB responsible for the fraction $\xi$ of the total optical depth $\tau_{0}$ is $\left[\lambda_{\xi}, \lambda_{\mathrm{th}}\right]$, where $\lambda_{\xi}$ and $\lambda_{\text {th }}$ are the abscissas of the points where the horizontal line $E=E_{0}$ intersects the corresponding level curve, and the threshold line, respectively. In Fig. 4 four levels for $\xi=10,50,90$, and $99 \%$, are shown. By definition, $\xi=0$ corresponds to the threshold boundary $E_{\mathrm{TeV}}=0.21 \lambda_{\mu}$. 
It is seen that at $E_{0}=17 \mathrm{TeV}$ approximately 50 per cent of the total optical depth $\tau_{0}$ is contributed by background photons with wavelengths longer than $50 \mu \mathrm{m}$. Therefore even the sharp cutoff of the CIB flux below $50 \mu \mathrm{m}$ cannot prevent the large optical depth of $17 \mathrm{TeV} \gamma$-rays, as far as we accept that the $60 \mu \mathrm{m}$ flux has a cosmological origin, and reflects the level of CIB at these wavelengths. This is the case of Model I for which the free path of $17 \mathrm{TeV}$ $\gamma$-rays is about $20 \mathrm{Mpc}$, and therefore for the distance to the source of $170 \mathrm{Mpc}\left(H_{0}=60 \mathrm{~km} \mathrm{~s}^{-1} \mathrm{Mpc}\right)$ the optical depth $\tau_{0} \approx 8.5$. The corresponding absorption factor is so large $\left(\exp (-8.5) \simeq 2 \times 10^{-4}\right)$ that the sharp file-up in the absorption-corrected spectrum becomes unavoidable.

Since the exponent in the absorption factor is very large, even a relatively small reduction of the optical depth $\tau_{0}$ may help to reduce significantly the effect of $\gamma$-ray attenuation. In particular, the Model III, which has a more realistic spectral shape at mid infrared wavelengths, and fits the FIR data above $100 \mu \mathrm{m}$, allows a bit larger (25 per cent or so) free paths at highest energy $\gamma$-rays. Although this makes the pile-up less pronounced, but still does not eliminate it altogether. Only the CIB Model II gives a comfortable, $E^{-2}$ type spectrum.

We may reduce the absorption effect furthermore, assuming a larger, although presently less favored value for the Hubble constant, $H_{0}=100 \mathrm{~km} \mathrm{~s}^{-1}$ Mpc. The latter makes smoother the pile-up for the Model I, and almost removes it for the Models III and IV. Therefore we may conclude that it is possible, in principle, to avoid the "disturbing" sharp turn-up in the intrinsic $\gamma$-ray spectrum of Mkn 501, if we adopt for CIB a model like the Model III, and assume a very large value for the Hubble constant.

Below we discuss, however, possible solutions which allow accommodation of both higher CIB fluxes and more realistic value for the Hubble constant.

\section{The effect of cascading in the intergalactic photon fields}

Generally, the propagation of high energy $\gamma$-rays trough a low frequency photon field cannot be reduced to the simple effect of $\gamma \gamma$ absorption. When a $\gamma$-ray is absorbed its energy in fact is not lost. The secondary electrons and positrons create new $\gamma$-rays via inverse Compton scattering; the second generation $\gamma$-rays produce new $\left(\mathrm{e}^{+}, \mathrm{e}^{-}\right)$ pairs, thus an electromagnetic cascade develops. Actually, in the intergalactic space this process is inevitable, and it may significantly contribute to the isotropic (extragalactic) $\gamma$-ray background radiation (Protheroe \& Stanev 1996; Coppi \& Aharonian 1997). Note that in the energy region of interest the $\gamma$-rays and electrons interact with photon fields of different origin. Although the energy density of the $2.7 \mathrm{~K}$ cosmic microwave background radiation (CMBR) well exceeds the density of other ("starlight" and "dust") components of the cosmic background radiation (see Fig. 1), because of the kinematic threshold of the reaction $\gamma \gamma \rightarrow \mathrm{e}^{+} \mathrm{e}^{-}$the $\gamma$-rays with energy less than several hundred $\mathrm{TeV}$ interact mostly with the infrared and optical background photons. The inverse Compton scattering of electrons does not have kinematic threshold, therefore the electrons interact predominantly with much denser CMBR.

For intrinsic $\gamma$-ray spectra harder than $E^{-2}$ extending to energies $E \geq 100 \mathrm{TeV}$, the cascade spectrum may well dominate over the primary $\gamma$-ray spectrum. On the other hand, the cascade spectrum typically has a standard shape which slightly depends on the primary $\gamma$-ray spectrum. This makes rather attractive the idea of interpretation of one of the most remarkable features of the $\mathrm{TeV}$ radiation of Mkn 501, namely the surprisingly stable spectral shape of the source in high state, despite dramatic variation of the absolute flux on timescales less than several hours (Aharonian et al. 1999b; Aharonian et al. 1999a). Moreover, as it was noticed by Aharonian et al. (1999b), the shape of the TeV spectrum of Mkn 501 given by Eq. (2) resembles the $\gamma$-ray spectrum formed during the cascade development in the photon field. Therefore, the quantitative study of this effect presents a definite interest.

The interpretation of the observed $\mathrm{TeV}$ emission from Mkn 501 in terms of the intergalactic cascade radiation requires an extremely low intergalactic magnetic field. Indeed, for the field exceeding $10^{-12} \mathrm{G}$, the $\gamma$-rays of the cascade origin should be observed in a form of an extended emission from a giant isotropic pair halo with an angular radius more than several degree (Aharonian et al. 1994). Both the detected angular size and the time variability of the $\mathrm{TeV}$ radiation from Mkn 501 excludes such a possibility (Aharonian et al. 1999a; Aharonian et al. 2001c). For lower magnetic fields, the cascade $\gamma$-rays penetrate almost on a straight line, thus the first argument based on the detected angular size becomes less stringent. Note however that for the distance to the source $d \geq 100 \mathrm{Mpc}$ even tiny deflections of the secondary (cascade) electrons by the intergalactic magnetic field would lead to non-negligible time delays of arriving $\gamma$-rays (Plaga 1995; Kronberg 1995):

$$
\Delta t_{B} \simeq 10(d / 170 \mathrm{Mpc})(E / 1 \mathrm{TeV})^{-2}\left(B / 10^{-18} \mathrm{G}\right)^{2} \mathrm{~h} .
$$

This would smear out the time variation of $\mathrm{TeV}$ fluxes observed on timescales less than several hours (Aharonian et al. 1999a), unless $B \leq 10^{-18} \mathrm{G}^{2}$.

For such a low intergalactic magnetic field, we have studied the spectral properties of electromagnetic cascades generated by the primary multi- $\mathrm{TeV}$ radiation of Mkn 501 in the intergalactic medium, using a fast numerical method based on the solution of adjoint cascade equations (Uchaikin \& Ryzhov 1988). Two processes have been taken into account: electron-positron pair production and inverse Compton scattering. The details of calculations will be published elsewhere.

\footnotetext{
2 Actually, due to non-zero $\left(\sim m_{\mathrm{e}} c^{2} / E\right)$ emission angles of the secondary products in the $\gamma \gamma \rightarrow \mathrm{e}^{+} \mathrm{e}^{-}$and $\mathrm{e} \gamma \rightarrow \mathrm{e} \gamma^{\prime}$ reactions, we should expect a non-negligible broadening, and consequently time delays of the cascade radiation even at the absence of magnetic field (Cheng \& Cheng 1996).
} 


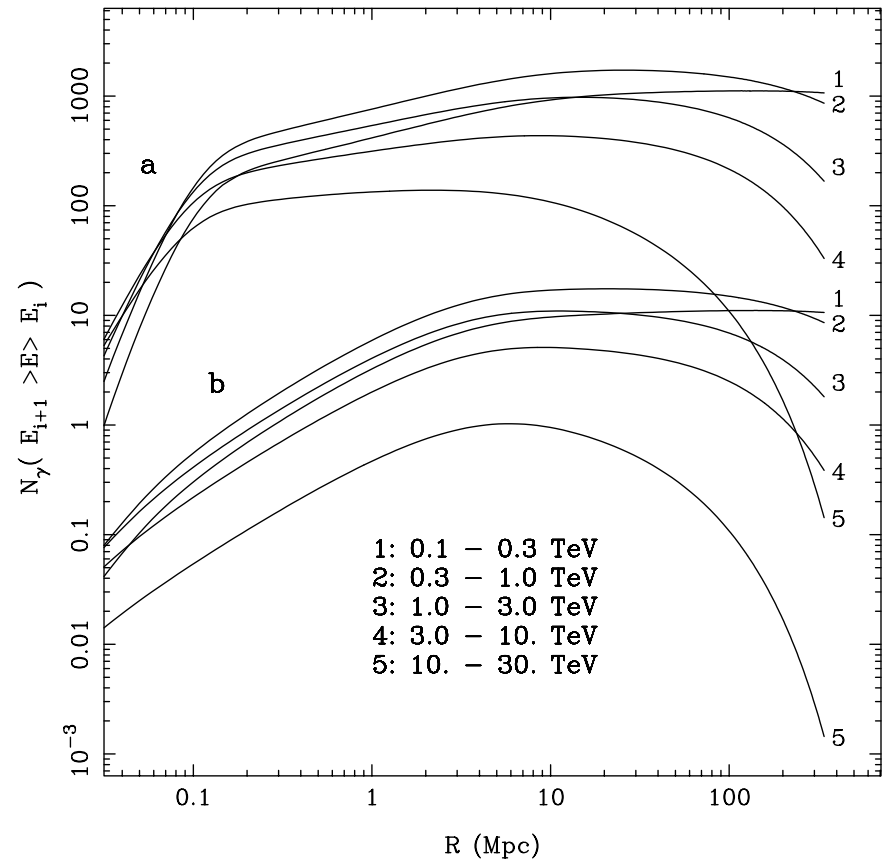

Fig. 5. The cascade development initiated by primary monoenergetic $\gamma$-rays with energy a) $E_{0}=10^{4} \mathrm{TeV}$ and b) $E_{0}=10^{2} \mathrm{TeV}$ for the CIB Model I. The curves show the number of cascade $\gamma$-rays in different energy intervals as a function of the penetration depth.

The idea behind the attempt to interpret $\mathrm{TeV}$ radiation of Mkn 501 by the intergalactic cascade is the following. The number of photons in a given energy band of the cascade spectrum may essentially exceed the number of $\gamma$-rays in the absorbed primary $\gamma$-ray spectrum. The $\gamma$-rays of highest energies, during their propagation trough the intergalactic photon fields, initiate cascades, which produce many lower energy photons, i.e. transfer the energy from the primary highest energy photons to lower energy $\gamma$-rays. Thus, the cascade somewhat "move" the source closer to the observer. In order to illustrate this effect, in Figs. 5 and 6 we show the number of cascade $\gamma$ rays in 5 energy bands as a function of the penetration depth. In the case of a cascade initiated by a monoenergetic primary $\gamma$-ray of energy $E_{0}$ (Fig. 5 ), the number of photons in all energy bands sharply increases and reaches its maximum at a distance $R_{*}$ which is determined approximately by the condition $\tau\left(E_{0}\right) \sim 1$ or $R_{*} \simeq L\left(E_{0}\right)$, where $L\left(E_{0}\right)$ is the mean free path of primary photons. Beyond $R_{*}$ the cascade develops more slowly. This stage is characterized by a competition between production and absorption processes. While the low energy (0.1-0.3 TeV) $\gamma$-rays continue to (slowly) grow, the number of higher energy $(10-30 \mathrm{TeV}) \gamma$-rays drops beyond $10 \mathrm{Mpc}$, the reason being the expiration of the "fuel", i.e. particles of sufficient energy, which could support further development of the cascade at these energies. It is interesting to note that the overall picture slightly depends on energy of the primary photon (compare curves in Fig. 5 calculated for $E_{0}=10^{4}$ and $10^{2} \mathrm{TeV}$ ). This explains why the spectrum

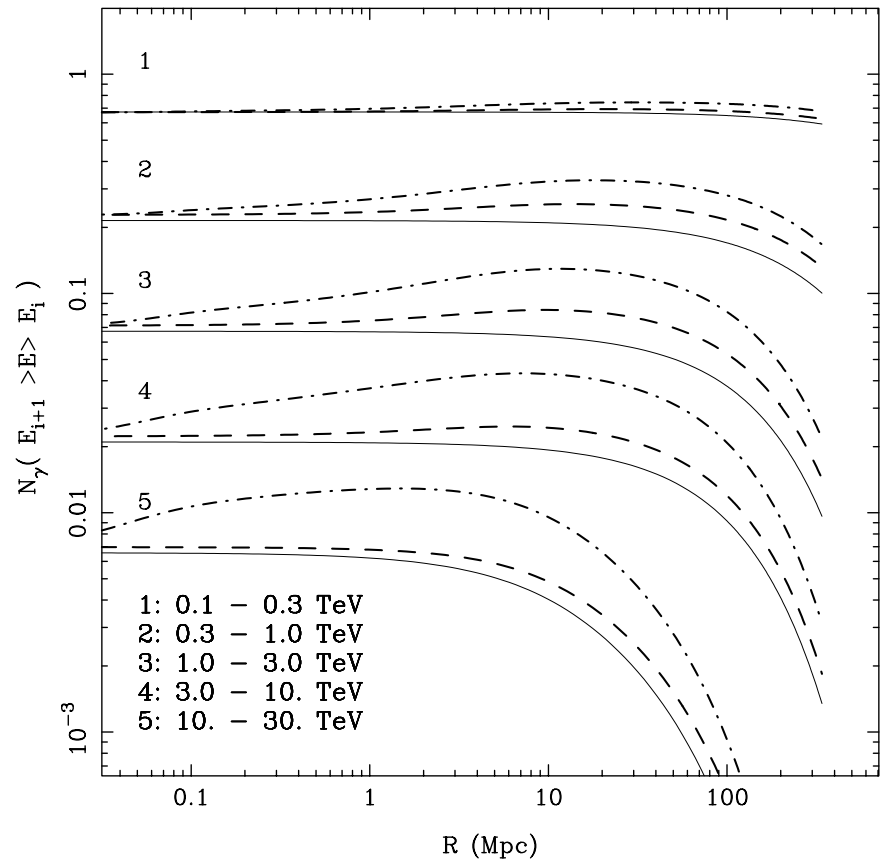

Fig. 6. The same as in Fig. 5, but for the power-law spectrum of primary $\gamma$-rays, $J_{0}(E) \propto E^{-2}$ extending to $E_{\max }=10^{2} \mathrm{TeV}$ (dashed lines) and $E_{\max }=10^{4} \mathrm{TeV}$ (dot-dashed lines). The solid lines show the evolution of the number of un-absorbed primary $\gamma$-rays, i.e. without the contribution of the secondary (cascade) component.

of $\gamma$-rays of the well-developed cascade becomes almost independent of the primary $\gamma$-ray spectrum.

The case of the cascade initiated by a broad-band spectrum of primary $\gamma$-ray photons is more complicated because the observer detects a mixture of the primary (un-absorbed) and secondary (cascade) $\gamma$-rays. Two example of cascades triggered by a $E^{-2}$ type primary $\gamma$-ray spectrum with $E_{\max }=10^{2} \mathrm{TeV}$ (dashed lines) and $E_{\text {max }}=10^{4} \mathrm{TeV}$ (dot-dashed lines) are shown in Fig. 6 . In order to demonstrate the photon excess caused by the cascade development, we show also the evolution of the number of photons calculated for the simple absorption effect, i.e. $J(E)=J_{0} \exp [-R / L(E)]$.

In Fig. 7 we show the spectra of cascade $\gamma$-rays from Mkn 501, calculated for the Hubble constant $H_{0}=$ $60 \mathrm{~km} \mathrm{~s}^{-1} / \mathrm{Mpc}$. The spectrum of the primary $\gamma$-radiation was assumed to be power-law $J_{0}(E) \propto E^{-\alpha}$ with $\alpha=1.9$. For softer source spectra $(\alpha>2)$, the cascade has a small impact on the resulting spectrum. For harder primary spectra with $\alpha<2$, the most power is radiated in the high energy range, and therefore the resulting spectrum is dominated by the "cascade" component.

For a narrow, e.g. Planckian type spectrum of target photons with temperature $T_{\mathrm{r}}$, the spectrum of welldeveloped cascade $\gamma$-rays has a standard power-law shape with photon index 1.5 at energies $E \ll E^{*} \simeq m_{\mathrm{e}}^{2} c^{4} / k T_{\mathrm{r}}$, and sharp cutoff beyond $E^{*}$. For a broad-band background photon field, the spectral shape of CIB plays an essential role in forming the cascade $\gamma$-ray spectrum, especially in 


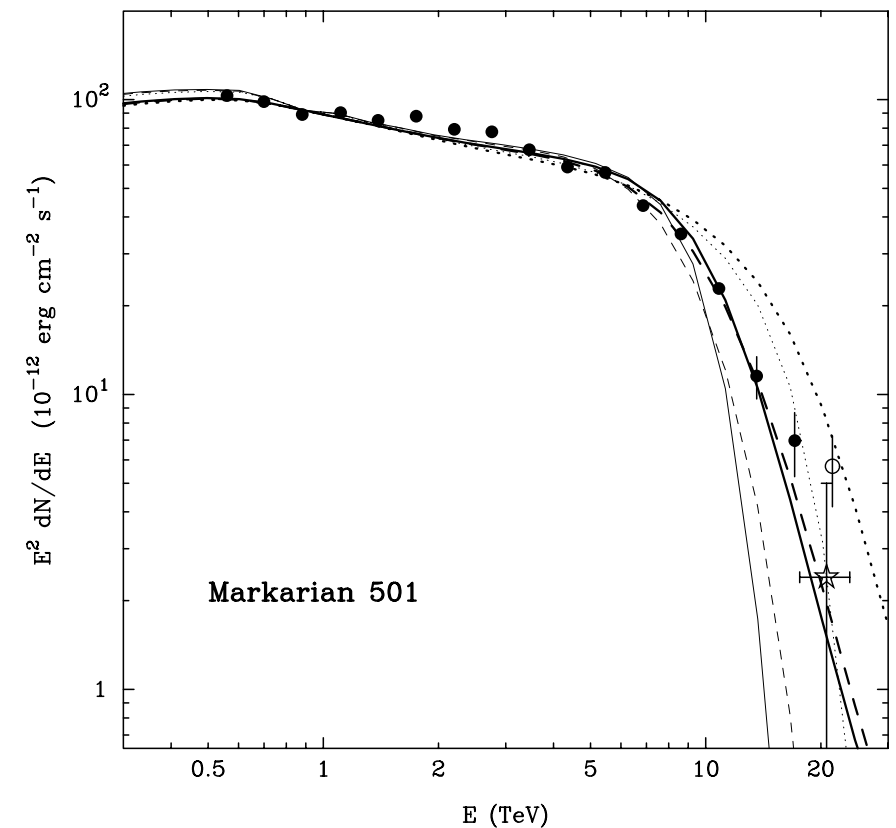

Fig. 7. Intergalactic cascade radiation spectra initiated by primary $\gamma$-rays from Mkn 501. The solid, dotted, and dashed lines correspond to the Model I, Model II, and Model III of CIB, respectively. The light and heavy lines represent the predicted cascade $\gamma$-ray fluxes before and after convolution with the Gaussian type energy spread function with $20 \%$ energy resolution.

the region of the cutoff. This can be seen in Fig. 7 where the $\gamma$-ray spectra are calculated for 3 different models of CIB presented in Fig. 1. For comparison, we show also the fluxes of Mkn 501 as measured by HEGRA during the high state of the source in 1997. The experimental points (filled circles up to $17 \mathrm{TeV}$ and an open circle at $\simeq 21 \mathrm{TeV}$ ) are obtained with an energy resolution of about $20 \%$. In addition, they contain $\approx 15 \%$ uncertainty on the energy scale throughout the entire interval from 0.5 to $20 \mathrm{TeV}$, and up to factor of 2 statistical and systematic uncertainties in flux estimates above $10 \mathrm{TeV}$ (Aharonian et al. 1999b)

Within these uncertainties, the cascade $\gamma$-ray spectrum corresponding to the CIB Model II (light dotted line) agrees with the HEGRA points, while the $\gamma$-ray spectra calculated for the CIB Models I and III (light solid and dashed curves, respectively) pass significantly below the measured fluxes at $E \geq 10 \mathrm{TeV}$ However, at these energies the spectra are very steep, therefore we must to take into account the limited instrumental energy resolution when comparing the theoretical predictions with the differential flux measurements. Namely, the experimental fluxes should be compared with the predictions after convolving the theoretical $\gamma$-ray spectra with the energy resolution function. In Fig. 7 the corresponding curves, obtained assuming Gaussian type instrumental energy spread function $G\left(E-E^{\prime}\right)=\exp \left(-\left(E-E^{\prime}\right)^{2} / 2 \sigma^{2}\right) /(\sqrt{2 \pi} \sigma)$ with constant $\sigma=E / 5$, i.e. $20 \%$ energy resolution, are shown by heavy solid (Model I), dotted (Model II) and dashed (Model III) lines. It is seen that after this procedure the

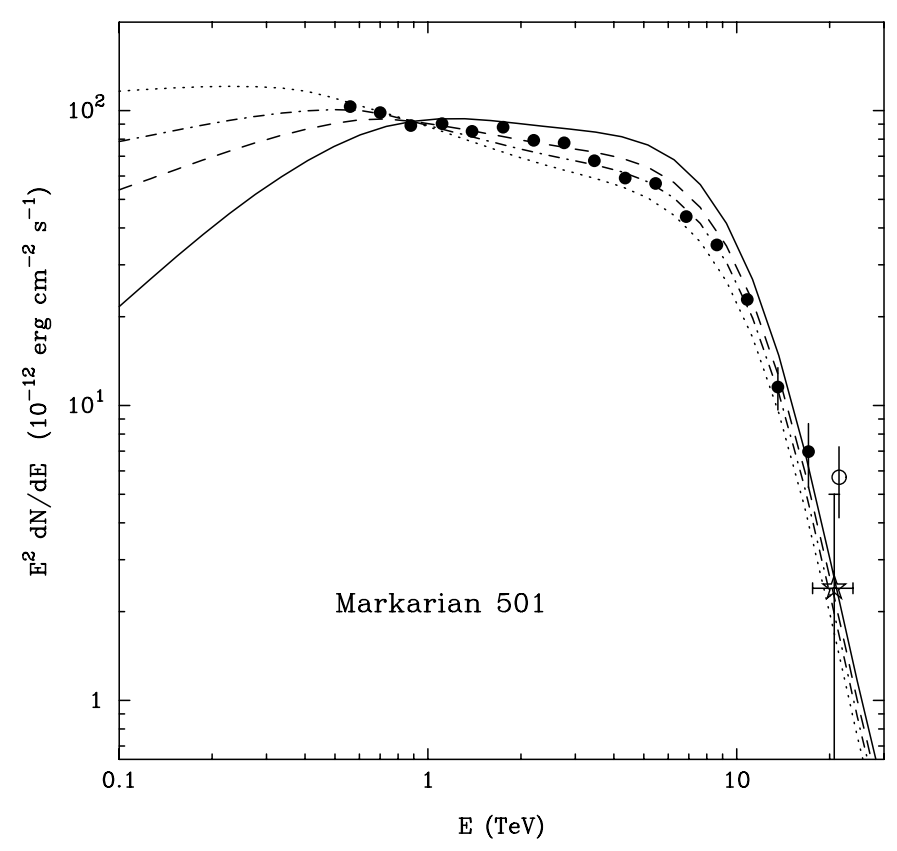

Fig. 8. Intergalactic cascade radiation spectra initiated by primary $\gamma$-rays from Mkn 501. The solid, dashed, dot-dashed and dotted curves are the cascade $\gamma$-ray spectra corresponding to the the photon indices of the primary $\gamma$-radiation $\alpha=1.5,1.8$, 1.9 and 2 , respectively. The curves are obtained after the convolution of the cascade $\gamma$-ray spectra with the Gaussian type energy spread function with $20 \%$ energy resolution. All curves are calculated for the CIB Model III.

$\gamma$-ray spectra corresponding to the CIB Models I and III quite satisfactorily fit the HEGRA points. At the same time the CIB Model II gives noticeably higher $\gamma$-ray fluxes.

The HEGRA stereoscopic system of telescopes has a potential to reduce the energy resolution down to $\sim 10 \%$ (Hofmann 2000). The recent re-analysis of the 1997 high state Mkn 501 data (Aharonian et al. 2001b) based on the improved energy reconstruction method confirmed the previous flux measurements, except the flux upper limit at $\simeq 21 \mathrm{TeV}$ which appeared by a factor of 2.5 below the previous flux estimate (the star and open circle in Fig. 7, respectively). Therefore the $20 \%$ energy resolution used for calculations presented in Fig. 7 should be considered as a rather conservative assumption. Nevertheless this seems to be, an appropriate assumption, at least for the purposes of this paper, given the lack of independent experimental methods for cross-calibration of the energy resolution of ground-based Cherenkov telescopes.

At high energies the spectrum of cascade photons weakly depends on the the power-law index $\alpha$ and the maximum energy $E_{\max }$ of primary $\gamma$-rays, provided that $\alpha \leq 2$ and $E_{\max } \gg 10^{2} \mathrm{TeV}$. In Fig. 8 four cascade $\gamma$-ray spectra are shown calculated for $\alpha=1.5,1.8,1.9,2$ and $E_{\max }=10^{3} \mathrm{TeV}$ and assuming $20 \%$ detector energy resolution. All spectra are calculated for the CIB model III. The photon indices of primary $\gamma$-rays $\alpha=1.8,1.9$, and 2 provide very similar fits for the HEGRA points throughout the entire energy interval from $0.5 \mathrm{TeV}$ to $20 \mathrm{TeV}$. 
They become distinguishable at low energies, but mainly because of the contributions of primary (un-absorbed) $\gamma$ rays which dominate below $1 \mathrm{TeV}$. The very hard primary spectrum with $\alpha=1.5$ results in a flatter cascade $\gamma$-ray spectrum compared to the measurements. On the other hand, the primary photon index $\alpha$ should not exceed 2.02.1 , otherwise the efficiency of the cascade contribution becomes very small. Hoverer, for the photon index of about 2 the primary spectrum has to extend beyond $100 \mathrm{TeV}$ in order to provide an adequate contribution from the cascade component.

\section{Gamma-radiation produced by ultrarelativistic unshocked jets}

The "intergalactic cascade" hypothesis requires extremely small intergalactic magnetic field of about $B \sim 10^{-18} \mathrm{G}$ and extension of the primary $\gamma$-ray spectrum well beyond $100 \mathrm{TeV}$. In the case of failure of any of these two conditions, the contribution of the secondary $\gamma$-rays produced in the intergalactic medium becomes negligible, and thus the attenuation of $\gamma$-rays is reduced to the simple absorption effect. Consequently, the absorption-corrected spectrum of $\gamma$-rays given by Eq. (1) adequately represent the intrinsic $\mathrm{TeV}$ spectrum of the source. If so, we may face a difficulty with the current common belief that the source spectrum of $\gamma$-rays should have a "decent" shape, i.e. be in accord with the predictions of conventional astrophysical scenarios suggested for blazars. Indeed, none of the models of blazars, in general, and of Mkn 501 in particular, allow the striking feature which appears unavoidably in the absorption-corrected spectrum of Mkn 501 above $10 \mathrm{TeV}$ (see Fig. 3), if we adopt that the reported high FIR fluxes are due to the truly extragalactic background radiation. All versions of both the electronic and hadronic models, suggested so far, predict smooth broad-band $\gamma$-ray spectra with characteristic (gradual or sharp) steepening above $10 \mathrm{TeV}$.

Formally, it is possible to reproduce a $\gamma$-ray spectrum with a sharp pile-up assuming very narrow features in the distribution of accelerated particles. It should be noticed in this regard that almost all particle acceleration models predict power-law distributions with high energy cut-offs. While the cutoff energy $E_{0}$ can be estimated, generally quite confidently, from the balance between the particle acceleration and the energy loss rates, the shape of the resulting particle spectrum in the cutoff region depends on many specific acceleration and energy dissipation mechanisms. Remarkably, even within the "ordinary" shock acceleration scenarios we may expect not only spectral cutoffs, but also pronounced pile-ups preceding the cutoffs (e.g. Malkov 1997; Melrose \& Crouch 1997; Protheroe \& Stanev 1999; Drury et al. 1999, see however Kirk 2001). Within the proton-synchrotron model of blazars, such a pile-up would result in a bump in the synchrotron $\mathrm{TeV}$ emission at $\simeq 0.3 \eta^{-1} D_{\mathrm{j}} \mathrm{TeV}$ (Aharonian 2000 ), where $\eta \geq 1$ is the so-called gyrofactor. Thus, the location of the bump at $E \geq 17 \mathrm{TeV}$ should require very

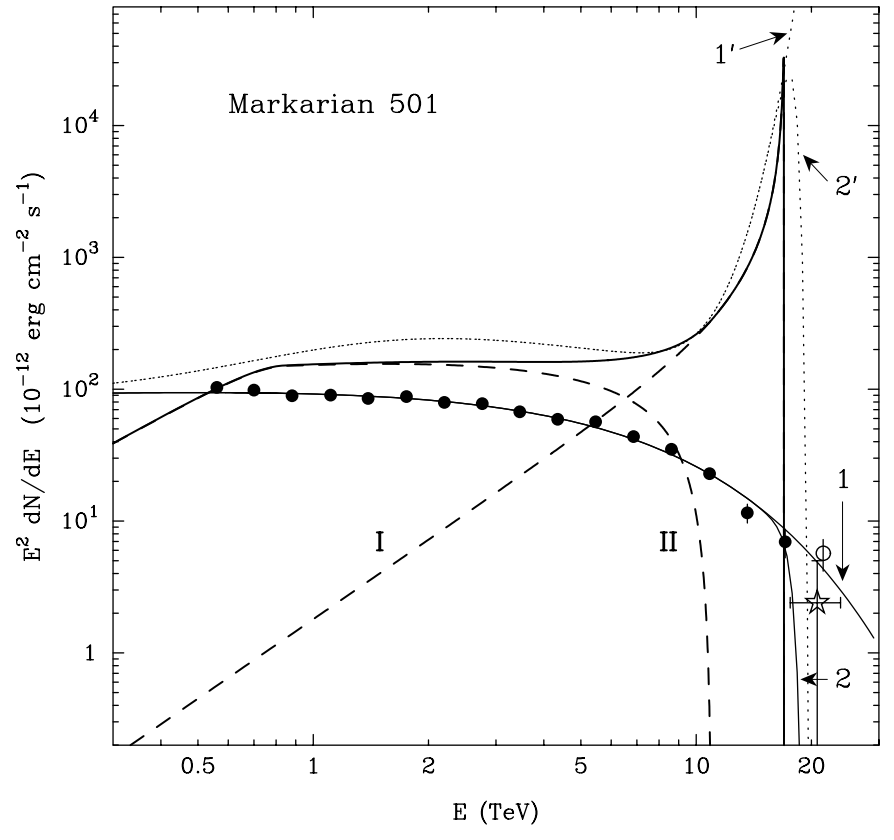

Fig. 9. Inverse Compton spectrum of cold unshocked ultrarelativistic jet with bulk motion Lorentz factor $\Gamma=3.33 \times 10^{7}$. The radiation component associated with comptonization of ambient optical photons with narrow spectral distribution is shown by the dashed line I. The IC spectrum on ambient photons with broad-band spectral distribution is shown by the dashed line II. The heavy solid line represents the superposition of these two components. Formally, the dashed line II could be treated as residual of the total $\mathrm{TeV}$ source emission after subtraction of the unshocked wind component I, and therefore can be referred to the IC radiation of blobs in shocked jet (see Fig. 11). Fits to the observed flux of Mkn 501 are shown by thin solid lines. Curve 1 corresponds to the fit given by Eq. (2) and curve 2 to the steepest possible spectrum above $17 \mathrm{TeV}$ based on the reanalysis of Mkn 501 HEGRA data (Aharonian et al. 2001b). Absorption-corrected spectrum of Mkn 501 for CIB Model I is shown by dotted lines $1^{\prime}$ and $2^{\prime}$ for the fits to the observed spectrum 1 and 2 respectively.

large Doppler factor $D_{\mathrm{j}} \geq 50 \eta$. In the leptonic models the pile-up in the electron spectrum would also result in corresponding features in the synchrotron X-ray and inverse Compton $\mathrm{TeV} \gamma$-ray spectra. The absorption-corrected spectra of Mkn 501 shown in Fig. 3 require very sharp pile-up in the electron spectrum. Whether such a pile-up could be formed in realistic particle acceleration models of small (sub-pc) jets of blazars is a question of future detailed studies.

In this paper we offer a different, non-acceleration scenario which postulates that the $\mathrm{TeV}$ radiation of Mkn 501 is a result of comptonization of the ambient low-frequency radiation by ultrarelativistic jet-like outflow with a Lorentz factor of the bulk motion of about $\Gamma \simeq(3-4) \times 10^{7}$.

The relativistically moving plasma outflows in forms of jets or winds, are common for many astrophysical phenomena on both galactic or extragalactic scales (see e.g. Mirabel 2000). Independent of the origin of these 
relativistic outflows, the concept of the jet seems to be the only successful approach to understand the complex features of nonthermal radiation of blazars, microquasars and GRBs. The Lorentz factor of such outflows could be extremely large. In particular, in the Crab Nebula the Lorentz-factor of the MHD wind is estimated between $10^{6}$ and $10^{7}$ (Rees \& Gunn 1974; Kundt \& Krotscheck 1980; Kennel \& Coroniti 1984). The conventional Lorentzfactors of jets in the inverse Compton models of $\gamma$-ray blazars, are rather modest, $\Gamma \sim 10$. However there are no apparent theoretical or observation arguments against the bulk motion with extreme Lorentz-factors (see e.g. discussion by Celotti et al. 1998 on Mkn 421). Meszaros \& Rees (1997) have shown that in the context of cosmological GRBs the magnetically dominated jet-like outflows from stellar mass black holes may attain extreme bulk Lorentz factors exceeding $10^{6}$. We are not aware of similar calculations (and conclusions) concerning the massive black holes - the engines of AGN. Therefore we will limit our discussion by postulating existence of an outflow in Mkn 501 which moves, at least at the initial stages of its propagation in the vicinity of the central black hole, with an extreme bulk Lorentz factor $\geq 10^{7}$. If true, it is almost obvious that the outflow should have MHD origin, the energy being extracted from the rotating black hole through e.g. the Blandford-Znajek type mechanism (see Blandford 2000 for a recent review). In proximity of the accretion disk, the outflow should be Poynting-flux dominated in order to avoid the Compton drag, and only at relatively large distances from the central object, where the photon density is significantly reduced, an essential fraction of the electromagnetic energy must be transfered to the kinetic energy of bulk motion.

From a cold relativistic outflow we do not expect significant synchrotron radiation. Indeed, although the energy of electrons in the frame of observer can be as large as $10 \mathrm{TeV}$, they move together with magnetic field, and thus they do not emit synchrotron photons. Nevertheless, the cold ultrarelativistic outflow could be visible through the inverse Compton $\gamma$-radiation of wind electrons. Apparently, in this scenario the dense target photon field is the second important ingredient for effective production of $\gamma$-rays. Remarkably, even in the case of relatively weak BL Lac objects there could be several important sources of infrared and optical emission within the inner sub-parsec region of the central source - IR emission from the dust torus, broad-line emission from fast moving clouds, starlight, etc. It is easy to show that the Compton optical depth $\tau_{\mathrm{C}}$ in this region could be as large as 1 (see e.g. Celotti et al. 1998). In fact, we have to introduce some additional conditions to avoid the destruction of the jet due to the Compton drag. On the other hand, since for $\tau_{\mathrm{C}} \leq 1$ the $\gamma$-ray luminosity is proportional to the product $L_{\mathrm{j}} \times \tau_{\mathrm{C}}$, the optical depth should be close to 1 in order to avoid assumptions which would require very large jet power $L_{\mathrm{j}}$. Obviously, the most favorable value for $\tau_{\mathrm{C}} \leq 1$ lies between 0.1 and 1 . Because of extremely large bulk Lorentz factor $\Gamma \geq 10^{7}$, the Compton scattering on the ambient optical photons and near-infrared photons proceeds in deep Klein-Nishina regime. Therefore the resulting $\gamma$-radiation should have a very narrow distribution with a maximum at the edge of the spectrum $E \approx E_{\mathrm{e}}=m_{\mathrm{e}} c^{2} \Gamma$. Meanwhile, the IC scattering on low-frequency target photon components, e.g. on far infrared photons of the dust torus, would still take place in the Thomson regime, and thus would produce a smooth broad-band $\gamma$-ray spectrum.

In Figs. 9 and 10 we demonstrate that the overall absorption-corrected spectrum of Mkn 501 can be satisfactorily interpreted in the terms of bulk inverse Compton emission of the jet, assuming a specific ambient radiation field consisting of two - narrow (Planckian) type radiation with temperature $k T \sim 1 \mathrm{eV}$ (curve I) and broader IR radiation, which formally could be presented in a powerlaw form $n(\epsilon) \propto \epsilon^{-m}$ (curve II). More specifically, the best fit for the CIB Model I is achieved for $k T=2 \mathrm{eV}$ and $m=1.8$, with the ratio of the energy densities of two components of about $r=w_{1} / w_{2}=163$. The CIB Model III requires similar, although slightly different parameters: $k T=0.2 \mathrm{eV}, m=1.8$, and $r=9.5$. In both cases the bulk Lorentz factor $\Gamma=3.33 \times 10^{7}$ is assumed.

The "Klein-Nishina" component, caused by inverse Compton scattering on the "hot" narrow photon distribution, is of prime interest because this component provides the most critical part of the spectrum - the pile-up. Moreover, we can omit the second ("Thomson") component of $\gamma$-radiation (for example assuming larger values for the ratio $r=w_{1} / w_{2}$ compared to the ratios used in Figs. 9 and 10). This should not be a problem for the model, because we may compensate this loss by assuming that the low $(E \leq 10 \mathrm{TeV})$ energy part of the $\gamma$-ray spectrum is formed at later stages of evolution of the jet, namely due to transformation of a fraction of the bulk kinetic energy of the jet into a random energy of relativistic electrons, e.g. in the form of so-called blobs (plasmons) moving with Lorentz factor of about 10. This can be realized through the acceleration of electrons by the terminal shock which may be created at interaction of the jet with the ambient matter (here again, we could see an interesting analogy with the Crab Nebula). Actually this is the stage which is treated in all conventional, SSC or external Compton models of X-ray and $\gamma$-ray emission of TeV blazars (Sikora \& Madejski 2001) with "standard" jet parameters like Doppler factor of about 10-30, and the magnetic field of about 0.1-1 Gauss (Inoue \& Takahara 1996; Bednarek \& Protheroe 1997; Tavecchio et al. 1998; Mastichiadis \& Kirk 1997; Krawczynski et al. 2000). The two-stage scenario of jet radiation is illustrated in Fig. 11.

\section{Discussion}

It is believed that the diffuse extragalactic infrared background radiation may have a dramatic impact on the models of $\mathrm{TeV}$ blazars. In particular, the recently reported high CIB fluxes, both at NIR and FIR bands, imply that we detect significantly absorbed $\mathrm{TeV}$ radiation even from 


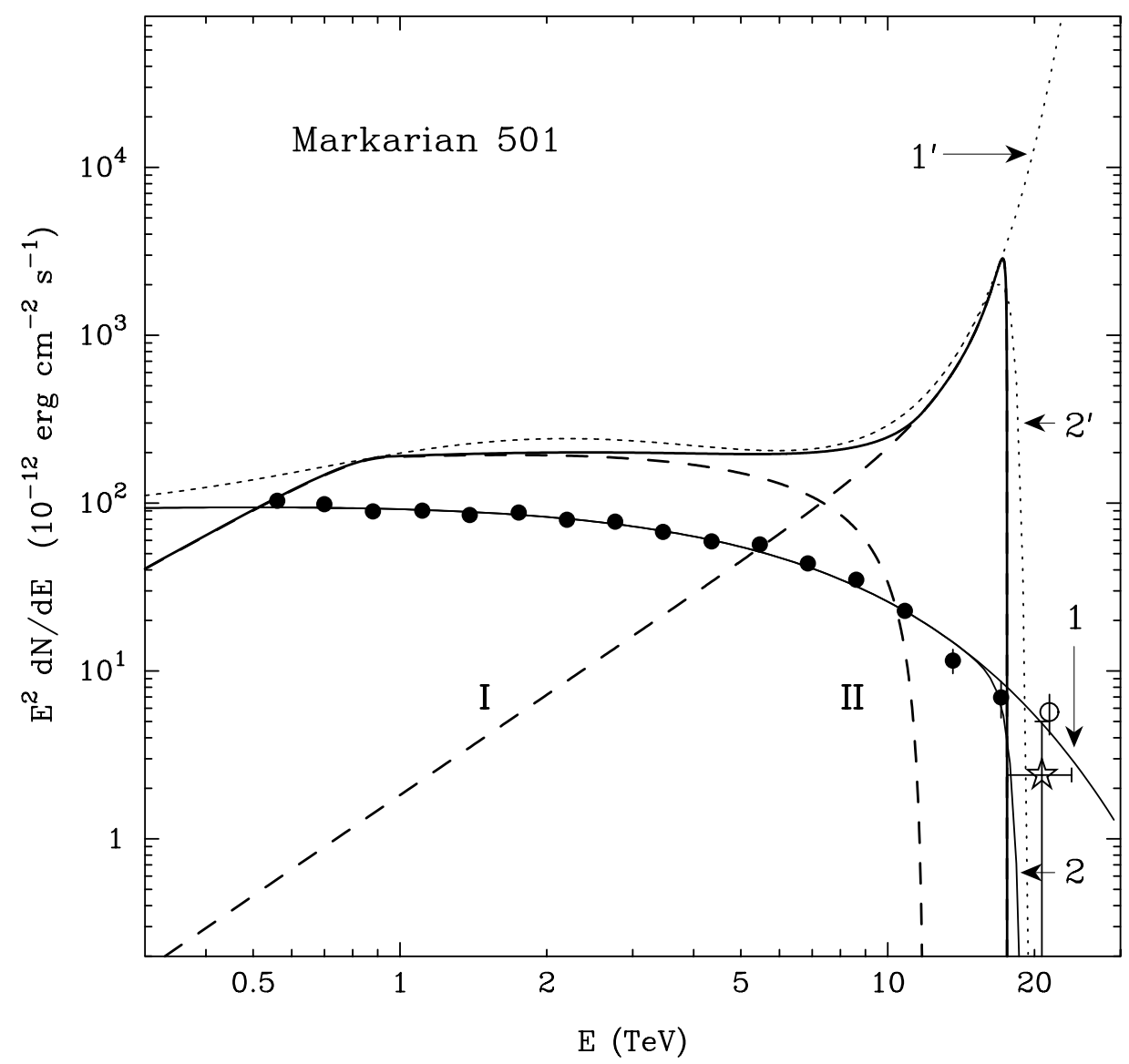

Fig. 10. The same as Fig. 9, but for CIB Model III.

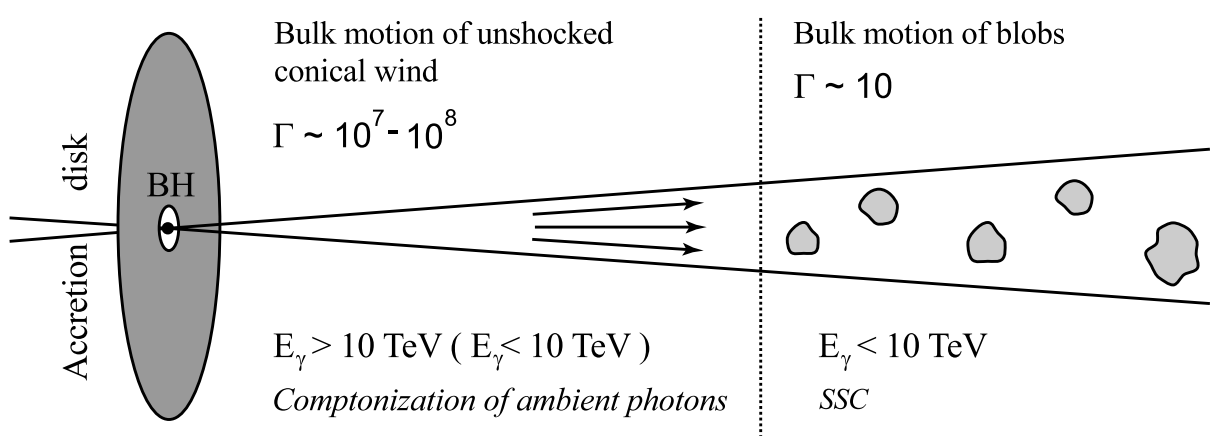

Fig. 11. Sketch of the two-stage $\gamma$-ray production scenario in Mkn 501. At the first stage, the observed highest energy $\gamma$-rays above $10 \mathrm{TeV}$ with a sharp spectral form ("pile-up") are produced due to comptonization of the ambient optical photons of energy $\sim 1-2 \mathrm{eV}$ by the cold ultrarelativistic jet with bulk motion Lorentz factor $\Gamma \sim 10^{7}-10^{8}$. Low energy $\gamma$-rays, $E_{\gamma} \leq 10 \mathrm{TeV}$ can be also produced due to comptonization of far infrared photons with broad spectral distribution. At the second stage, relatively low energy $\left(E_{\gamma} \leq 10 \mathrm{TeV}\right) \gamma$-rays are produced in the blobs moving with Lorentz factor of about $\Gamma \sim 10$ in accordance with the standard SSC or external IC models.

relatively nearby objects like Mkn 421 and Mkn 501. Due to the energy-dependent mean free path of $\gamma$-rays in the intergalactic medium, the detected spectra of $\mathrm{TeV}$ emission from extragalactic objects at cosmological distances significantly deviates from the source spectra. The intergalactic absorption does not simply imply spectral cutoffs, but rather modulates the primary spectrum from sub-TeV to multi-TeV energies. For some specific CIB models, the "high-state" TeV spectrum of Mkn 501 after correction for the intergalactic absorption becomes rather flat (Fig. 3), $\mathrm{d} N / \mathrm{d} E \propto E^{-2}$ (e.g. Coppi \& Aharonian 1999a; Konopelko et al. 1999) or even harder (Guy et al. 2000; Renault et al. 2001; de Jager \& Stecker 2002), if the CIB density of the mid-infrared "valley" exceeds $\nu F_{\nu} \geq 4 \mathrm{nW} / \mathrm{m}^{2} \mathrm{sr}$ (Aharonian et al. 1999b). Although formally such spectra can be described within the conventional SSC models, the detailed treatment of the problem within the one-zone SSC model requires quite uncomfortable jet parameters, in particular very large Doppler factor $\delta_{\mathrm{j}} \geq 100$, and very weak 
magnetic field $B \leq 0.01 \mathrm{G}$. If so, this perhaps would require a serious consideration alternative models for $\mathrm{TeV}$ emission like the proton-synchrotron model which can satisfactorily fit the data of Mkn 501 and Mkn 421 even for quite high CIB fluxes (Aharonian 2000).

However all these models fail to explain the sharp pileup which appears at the end of the "reconstructed" spectrum of Mkn 501, if the reported FIR fluxes correctly describe the level of the diffuse cosmic background radiation. Remarkably, such a pile-up is unavoidable not only due to the reported extremely high flux at $60 \mu \mathrm{m}$, which obviously needs further confirmation, but also, albeit in a less distinct form, due to the fluxes at longer, $\lambda \geq 100 \mu \mathrm{m}$ wavelengths reported by several independent groups. To avoid such an unusual shape of $\gamma$-radiation, recently several dramatic assumptions have been proposed concerning the nature of the detected signals or the violation of the Lorenz-invariance at very high energies.

The main objective of this work was the attempt to investigate other possible ways to overcome the "IR background- $\mathrm{TeV}$ gamma-ray crisis". In particular, we studied two possibilities: (i) assuming that the observed $\mathrm{TeV}$ spectrum of $\gamma$-rays from Mkn 501 is formed due to the cascade initiated by primary $\gamma$-rays in the intergalactic medium, and (ii) assuming that the detected highest energy $\gamma$-rays are result of bulk-motion comptonization of the ambient optical photons by the ultrarelativistic unshocked conical wind emerging from the central source.

\subsection{Rectilinear intergalactic cascade radiation}

The electromagnetic cascades in the extragalactic photon fields result in a non-negligible increase of the effective propagation path of $\gamma$-rays. Remarkably, the results presented in Sect. 3 show that the cascade $\gamma$-ray spectra can quite well fit the time-averaged high state spectrum of Mkn 501. An important feature of the well-developed cascade radiation is its standard spectral shape which is determined by the propagation effects and weakly depends on the details of the primary radiation of the central source. Thus, this hypothesis could give a natural explanation for the quite stable spectral shape of the source in high state, despite dramatic variation of the absolute flux on timescales less than several hours (Aharonian et al. 1999b; Aharonian et al. 1999a). Note that the spectral change during the strong April 16, 1997 flare reported by the CAT collaboration (Djannati-Atar et al. 1999), as well as the noticeable steepening of the spectrum in a low state of Mkn 501, found by the HEGRA collaboration (Sambruna et al. 2000; Aharonian et al. 2001a) do not contradict to this statement. These effects, in fact, could be caused by variations of the ratio of the "cascade" component to the overall ("unabsorbed" plus "cascade") flux of $\gamma$-rays due to, for example, reduction of the maximum energy in the primary $\gamma$-ray spectrum at the low or quiescent states.
The "intergalactic cascade" hypothesis requires extremely small, at least in the direction of Mkn 501, intergalactic magnetic fields, in order to avoid the significant time delays. Even a tiny intergalactic magnetic field of about $10^{-18} \mathrm{G}$ should lead to delays of arrival of $\mathrm{TeV}$ $\gamma$-rays, compared to the associated low energy photons, by more than several days. This obviously contradicts the observed X/TeV correlations (e.g. Pian et al. 1998; Catanese et al. 1997; Krawczynski et al. 2000). Although indeed very speculative, formally such small fields cannot be excluded, especially if we take into account that the typical scale of the so-called intergalactic voids, where the magnetic field could be arbitrarily small, is estimated as $\sim 120\left(H_{0} / 100 \mathrm{~km} / \mathrm{s} / \mathrm{Mpc}\right)^{-1} \mathrm{Mpc}$ (Einasto 2000), i.e. quite comparable to the distance to Mkn 501.

The second condition for realization of this model the extension of the primary spectrum of Mkn 501 out to $100 \mathrm{TeV}-$ also is a very robust requirement. Although the discussion of possible mechanisms of production of such energetic photons is beyond the framework of this paper, we note that the condition of $E_{\max } \gg 100 \mathrm{TeV}$ cannot be easily accommodated by the conventional (especially, leptonic) models of high energy radiation of Mkn 501.

Apparently, the "intergalactic cascade" hypothesis which simply postulates very low intergalactic magnetic fields and very energetic primary $\gamma$-radiation of the source, cannot provide conclusive predictions for the time correlations of $\mathrm{TeV} \gamma$-rays with nonthermal radiation components at other energy bands. At the same time, this hypothesis allows robust calculations of spectral characteristics of $\gamma$-radiation at different depths of the cascade development. Therefore the most straightforward (and perhaps relatively easy) inspection of this hypothesis would be the study of spectral characteristics of $\mathrm{TeV}$ $\gamma$-rays detected from sources with different redshifts $z$.

\subsection{Inverse Compton radiation of ultrarelativistic cold outflow}

Although the assumption about the existence of a cold ultrarelativistic outflow with an extreme Lorentz factor of bulk motion $\Gamma \sim 3.5 \times 10^{7}$ is somewhat unusual and perhaps even provocative (at least it has not been discussed in the literature before), it cannot be a priori ruled out. A similar scenario most probably takes place, although on significantly smaller scales, in environments of pulsars. The rotation-powered pulsars eject plasma in the form of relativistic winds which carry off bulk of the rotational energy. At a distance $d \leq 1 \mathrm{pc}$ the wind is terminated by a strong standing reverse shock which accelerates particles and randomizes their pitch angles. This results in formation of strong synchrotron and inverse Compton nebulae. On the other hand, it is generally believed that the region between the pulsar magnetosphere and the shock is invisible because the electrons move together with magnetic field and thus do not emit synchrotron radiation. However, recently it has been argued that such winds could be 
directly observed through their inverse Compton emission, the low-frequency seed photons for comptonization being provided by the neutron star in the case of radiopulsars like Crab or Vela (Bogovalov \& Aharonian 2000) or by the optical companion star in the case of binary pulsars (Ball \& Kirk 2000). If such a cold ultrarelativistic conical wind can indeed be produced in the proximity of the central rotating black hole, because of possible existence of dense photons fields in the inner sub-parsec region, it could be a very powerful emitter of inverse Compton $\gamma$-radiation. The later however would not be accompanied by noticeable synchrotron radiation.

The possibility to disentangle the multi-TeV emission with a characteristic sharp pile-up at the very end of the spectrum, $E_{\gamma} \simeq m_{\mathrm{e}} c^{2} \Gamma$, associated with the unshocked jet, from the sub-10 TeV emission associated with the shocked structures (e.g. blobs) in the jet (see Fig. 11), not only may solve the "IR background-TeV gamma-ray crisis" but also would allow more relaxed parameter space for interpretation of X-rays and the remaining low energy $(\leq 10 \mathrm{TeV}) \gamma$-rays within the conventional SSC scenario. Consequently, this offers more options for interpretation of X-ray/TeV $\gamma$-ray correlations both on small $(t \leq$ several hours) and large (weeks or more) timescales. If the overall TeV radiation of Mkn 501 indeed consists of two, unshocked and shocked jet radiation components (curves I and II in Figs. 9, 10), we may expect essentially different time behaviors of these radiation components. In particular, the "unshocked jet" $\geq 10 \mathrm{TeV}$ radiation should arrive earlier than the SSC components of radiation consisting of synchrotron X-rays and sub-10 TeV $\gamma$-rays. Generally, all principal parameters of blobs like the magnetic field $B$, the maximum energy of electrons $E_{\max }$, the radius of the blob, etc., which define the spectra and absolute fluxes of synchrotron X-rays and IC TeV $\gamma$-rays, may dramatically evolve in time (Coppi \& Aharonian 1999a). Therefore we should expect strong $\mathrm{X} / \mathrm{TeV}$ correlations, especially during strong flares of the source. These correlations may be realized in quite different forms, i.e. it could vary from flare to flare depending on the time-evolution of specific parameters of blobs. At the same time the $\mathrm{IC}$ radiation of the unshocked jet significantly depends only on the Lorentz factor of the jet $\Gamma$. If the latter during short flares remains unchanged, we should expect rather stable spectral shape of the $\geq 10 \mathrm{TeV}$ emission, despite strong variations of the $\mathrm{SSC}$ radiation (X- and $\gamma$-ray) components. However, it should be noticed that because of possible external broadband infrared radiation fields, the unshocked jet radiation component may significantly contribute to the low energy $\mathrm{TeV}$ radiation as well (see Sect. 4). If so, it would enhance correlations between high and low energy bands of $\mathrm{TeV}$ radiation, On the other hand, it would reduce and make more complicated correlations between the low energy $\mathrm{TeV} \gamma$-rays and synchrotron X-rays.

On larger time-scales, e.g. in a low state of the source which could last weeks or months, both the particle density and the Lorentz factor of the unshocked jet may be smaller than in high states. This would not only reduce the luminosity of all components of nonthermal radiation, but also would make softer the overall $\gamma$-ray spectrum. Apparently, the pile-up associated with the radiation of cold wind would be shifted towards lower energies. Although its intensity could be significantly weakened, nevertheless the search for a relevant spectral feature seems to be an interesting opportunity, especially if we take into account that at these energies the intergalactic absorption effect also becomes weaker. Because of low photon statistics such studies can be effectively performed only by next generation ground-based detectors.

The straightforward proof of the suggested model would be direct detection of $\mathrm{TeV} \gamma$-ray spectra with characteristic sharp high energy pile-up. Apparently this requires nearby $\mathrm{TeV}$ sources with $d \ll 100 \mathrm{Mpc}$, thus the main fraction of $\geq 10 \mathrm{TeV} \gamma$-rays would arrive without significant intergalactic absorption. Unfortunately, all currently known BL Lacs are located beyond $100 \mathrm{Mpc}$. This significantly limits the chances for direct detection of sharp pile-ups in the spectra of BL Lacs or other type of blazars, unless the initial Lorentz factors of ultrarelativistic outflows in some objects do not exceed $\Gamma \leq 10^{7}$.

Important tests of the suggested two stage (pre-shock plus post shock) scenario of the TeV radiation of jets can be provided also by the search for correlations (or lack of such correlations) of high energy (multi-TeV) $\gamma$-radiation with both the low energy (e.g. 1-3 TeV) $\gamma$-rays and synchrotron X-rays. The low statistics of (heavily absorbed) $\gamma$-rays above $10 \mathrm{TeV}$ makes the search for such correlations rather difficult, and requires ground-based instruments with very large, $\gg 10^{5} \mathrm{~m}^{2}$ detection areas in this energy domain. The new generation imaging Cherenkov telescope arrays like CANGAROO-3, H.E.S.S. and VERITAS should be able, hopefully, to perform such correlation studies.

Acknowledgements. We thank Paolo Coppi, Julien Guy and Henric Krawczynski for fruitful discussions. AP and AT thank Max-Planck-Institut für Kernphysik for its generous hospitality. We are very grateful the anonymous referee for her/his useful suggestions which significantly improved the paper. In particular, the referee called our attention to the fact that the convolution of the predicted cascade spectrum with the instrumental energy spread function should improve the agreement with the observed $\mathrm{TeV}$ spectrum of Mkn 501.

\section{References}

Aharonian, F. A. 2000, New Astron., 5, 377

Aharonian, F. A., Akhperjanian, A. G., Barrio, J. A., et al. 1999a, A\&A, 349, 29

Aharonian, F. A., Akhperjanian, A. G., Barrio, J. A., et al. 1999b, A\&A, 349, 11

Aharonian, F. A., Akhperjanian, A. G., Barrio, J. A., et al. 2000, ApJ, 543, L39

Aharonian, F. A., Akhperjanian, A. G., Barrio, J. A., et al. 2001a, ApJ, 546, 898

Aharonian, F. A., Akhperjanian, A. G., Barrio, J. A., et al. 2001b, A\&A, 366, 62

Aharonian, F. A., Akhperjanian, A. G., Barrio, J. A., et al. 2001c, A\&A, 366, 746 
Aharonian, F. A., Coppi, P. S., \& Voelk, H. J. 1994, ApJ, 423, L5

Aloisio, R., Blasi, P., Ghia, P. L., \& Grillo, A. F. 2000, Phys. Rev. D, 62, 053010

Amelino-Camelia, G., \& Piran, T. 2001, Phys. Lett. B, 497, 265

Arp, H. C., Narlikar, J. V., \& Radecke, H. 1997, Astroparticle Phys., 6, 387

Ball, L., \& Kirk, J. G. 2000, Astroparticle Phys., 12, 335

Bednarek, W., \& Protheroe, R. J. 1997, MNRAS, 292, 646

Bernstein, R. A. 1999, in Low Surface Brightness Universe, ed. J. I. Davies, C. Impey, \& S. Phillipps, ASP Conf. Ser., 170

Biviano, A., Metcalfe, L., Altieri, B., et al. 2000, in Clustering at High Redshift, ASP Conf. Ser., 200, 101 [astro-ph/9910314]

Blandford, R. D. 2000 [astro-ph/0001499]

Bogovalov, S. V., \& Aharonian, F. A. 2000, MNRAS, 313, 504

Catanese, M., Bradbury, S. M., Breslin, A. C., et al. 1997, ApJ, 487, L143

Celotti, A., Fabian, A. C., \& Rees, M. J. 1998, MNRAS, 293, 239

Cheng, L. X., \& Cheng, K. S. 1996, ApJ, 459, L79

Coleman, S., \& Glashow, S. L. 1999, Phys. Rev. D, 59, 116008

Coppi, P. S., \& Aharonian, F. A. 1997, ApJ, 487, L9

Coppi, P. S., \& Aharonian, F. A. 1999a, ApJ, 521, L33

Coppi, P. S., \& Aharonian, F. A. 1999b, Astroparticle Phys., 11,35

de Jager, O., \& Stecker, F. 2002, ApJ, 566, 738

Djannati-Atal, A., Piron, F., Barrau, A., et al. 1999, A\&A, 350,17

Drury, L. O., Duffy, P., Eichler, D., \& Mastichiadis, A. 1999, A\&A, 347, 370

Dwek, E., \& Arendt, R. G. 1998, ApJ, 508, L9

Dwek, E., Arendt, R. G., Hauser, M. G., et al. 1998, ApJ, 508, 106

Einasto, J. 2000, in 9th Marcel Grossmann Meeting on relativistic astrophysics, ed. R. Ruffini (World Scientific), in press [astro-ph/0011334]

Finkbeiner, D. P., Davis, M., \& Schlegel, D. J. 2000, ApJ, 544, 81

Fixsen, D. J., Dwek, E., Mather, J. C., Bennett, C. L., \& Shafer, R. A. 1998, ApJ, 508, 123

Franceschini, A., Aussel, H., Cesarsky, C., Elbaz, D., \& Fadda, D. 2001, A\&A, 508, 1

Gorjian, V., Wright, E. L., \& Chary, R. R. 2000, ApJ, 536, 550

Gould, R. J., \& Schreder, G. P. 1967, Phys. Rev., 155, 1408

Guy, J., Renault, C., Aharonian, F. A., Rivoal, M., \& Tavernet, J. 2000, A\&A, 359, 419

Hacking, P. B., \& Soifer, B. T. 1991, ApJ, 367, L49

Harwit, M. 1999, ApJ, 510, L83

Harwit, M., Protheroe, R. J., \& Biermann, P. L. 1999, ApJ, 524, L91

Hauser, M. G., Arendt, R. G., Kelsall, T., et al. 1998, ApJ, 508, 25

Hauser, M. G., \& Dwek, E. 2001, ARA\&A, 39, 249

Hofmann, W. 2000, Astroparticle Phys., 12, 207

Hoyle, F., \& Burbidge, G. 1996, A\&A, 309, 335

Inoue, S., \& Takahara, F. 1996, ApJ, 463, 555

Kennel, C. F., \& Coroniti, F. V. 1984, ApJ, 283, 694

Kifune, T. 1999, ApJ, 518, L21

Kirk, J. G. 2001, in Proc. of ICRC 2001, in press

Kluźniak, W. 1999, Astroparticle Phys., 11, 117

Konopelko, A. K., Kirk, J. G., Stecker, F. W., \& Mastichiadis, A. 1999, ApJ, 518, L13
Krawczynski, H., Coppi, P. S., Maccarone, T., \& Aharonian, F. A. 2000, A\&A, 353, 97

Kronberg, P. P. 1995, Nature, 374, 404

Kundt, W., \& Krotscheck, E. 1980, A\&A, 83, 1

Lagache, G., Abergel, A., Boulanger, F., Désert, F. X., \& Puget, J. 1999, A\&A, 344, 322

Malkan, M. A., \& Stecker, F. W. 2001, ApJ, 555, 641

Malkov, M. A. 1997, ApJ, 491, 584

Mastichiadis, A., \& Kirk, J. G. 1997, A\&A, 320, 19

Melrose, D. B., \& Crouch, A. 1997, Publications of the Astronomical Society of Australia, 14, 251

Meszaros, P., \& Rees, M. J. 1997, ApJ, 482, L29

Mirabel, I. F. 2000 [astro-ph/0005591]

Nikishov, A. I. 1962, Sov. Phys. JETP, 14, 393

Pei, Y. C., Fall, S. M., \& Hauser, M. G. 1999, ApJ, 522, 604

Pian, E., Vacanti, G., Tagliaferri, G., et al. 1998, ApJ, 492, L17

Plaga, R. 1995, Nature, 374, 430

Pozzetti, L., \& Madau, P. 2000, in The Extragalactic Infrared Background and its Cosmological Implications, ed. M. Harwit, \& M. G. Hauser, IAU Symp. 204, in press [astro-ph/0011359]

Pozzetti, L., Madau, P., Zamorani, G., Ferguson, H. C., \& Bruzual, A. G. 1998, MNRAS, 298, 1133

Primack, J. R., Bullock, J. S., Somerville, R. S., \& MacMinn, D. 1999, Astroparticle Phys., 11, 93

Primack, J. R., Somerville, R. S., Bullock, J. S., \& Devriendt, J. E. G. 2001, in International Symp. on Gamma-Ray Astronomy, Heidelberg, June 2000, ed. F. Aharonian, \& H. Völk (AIP), AIP Conf. Proc., 558, 463 [astro-ph/0011475]

Protheroe, R. J., \& Meyer, H. 2000, Phys. Lett. B, 493, 1

Protheroe, R. J., \& Stanev, T. 1996, Phys. Rev. Lett., 77, 3708

Protheroe, R. J., \& Stanev, T. 1999, Astroparticle Phys., 10, 185

Rees, M. J., \& Gunn, J. E. 1974, MNRAS, 167, 1

Renault, C., Barrau, A., Lagache, G., \& Puget, J.-L. 2001, A\&A, 371, 771

Sambruna, R. M., Aharonian, F. A., Krawczynski, H., et al. 2000, ApJ, 538, 127

Schlegel, D. J., Finkbeiner, D. P., \& Davis, M. 1998, ApJ, 500, 525

Sikora, M., \& Madejski, G. 2001, in International Symp. on Gamma-Ray Astronomy, Heidelberg, June 2000, ed. F. Aharonian, \& H. Völk (AIP), AIP Conf. Proc., 558, 275 [astro-ph/0101382]

Silk, J., \& Devriendt, J. 2000, in The Extragalactic Infrared Background and its Cosmological Implications, ed. M. Harwit, \& M. G. Hauser, IAU Symp. 204, in press [astro-ph/0010460]

Stecker, F. 2000, in The Extragalactic Infrared Background and its Cosmological Implications, ed. M. Harwit, \& M. G. Hauser, IAU Symp. 204, in press [astro-ph/0010015]

Stecker, F. W., de Jager, O. C., \& Salamon, M. H. 1992, ApJ, 390, L49

Tavecchio, F., Maraschi, L., \& Ghisellini, G. 1998, ApJ, 509, 608

Uchaikin, V. V., \& Ryzhov, V. V. 1988, Stochastic theory of transport of high energy particles (Nauka, Novosibirsk), in Russian

Vassiliev, V. V. 2000, Astroparticle Phys., 12, 217

Wright, E. L., \& Johnson, B. D. 2001, ApJ, submitted [astro-ph/0107205] 